Revue scientifique sur la conception et l'aménagement de l'espace

\title{
Le paysage périurbain, outil de coopération entre métropoles européennes alpines
}

The Peri-Urban Landscape, A Tool for Cooperation between European Alpine metropolitan Regions

\section{Aurore Meyfroidt}

\section{(2) OpenEdition} Journals

Édition électronique

URL : https://journals.openedition.org/paysage/393

DOI : $10.4000 /$ paysage.393

ISSN : 1969-6124

Éditeur :

École nationale supérieure du paysage de Versailles-Marseille, Institut national des sciences appliquées Centre Val de Loire - École de la nature et du paysage, École nationale supérieure d'architecture et de paysage de Bordeaux, École nationale supérieure d'architecture et de paysage de Lille, Agrocampus Angers

\section{Référence électronique}

Aurore Meyfroidt, «Le paysage périurbain, outil de coopération entre métropoles européennes alpines », Projets de paysage [En ligne], 19 | 2018, mis en ligne le 01 décembre 2018, consulté le 21 septembre 2021. URL : http://journals.openedition.org/paysage/393 ; DOI : https://doi.org/10.4000/ paysage.393

Ce document a été généré automatiquement le 21 septembre 2021.

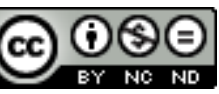

La revue Projets de paysage est mise à disposition selon les termes de la Licence Creative Commons Attribution - Pas d'Utilisation Commerciale - Pas de Modification 4.0 International. 


\title{
Le paysage périurbain, outil de coopération entre métropoles européennes alpines
}

\author{
The Peri-Urban Landscape, A Tool for Cooperation between European Alpine \\ metropolitan Regions
}

Aurore Meyfroidt

1 Au-delà d'une vision sectorielle liée à la préservation de la biodiversité et des écosystèmes, le paysage est moteur de projets de territoires et interroge l'action publique à plusieurs niveaux (Guisepelli et Fleury, 2007; Moquay et al., 2007). Il permet d'agir sur le territoire mais surtout de mobiliser les acteurs et il est alors considéré soit comme argument d'intérêt général cadrant l'action, soit comme outil saisi par certains acteurs pour défendre leur légitimité à agir (Sgard, 2010). Il est à la fois objet et matrice de l'action publique sur les territoires mais son caractère opératoire dans la planification et son degré d'inclusivité (en comparaison au développement durable par exemple) font encore débat.

2 Le caractère multi-acteur et multiniveau de la gouvernance des régions métropolitaines interroge les modalités d'action publique sur et par le paysage. L'Union européenne (UE), en lien avec la mise en place de la politique de cohésion, est un acteur important dans la production de connaissances et de politiques sur les espaces métropolitains, et le paysage est touché au même titre que d'autres secteurs: la mise en place de la Convention européenne du paysage (2000) puis de la Stratégie européenne sur les infrastructures vertes (2013) véhicule, pour la première, une conception du paysage ordinaire, support de biodiversité tout en encourageant, pour la seconde, la constitution d'un réseau transeuropéen d'espaces naturels, agricoles et forestiers, à l'intérieur et autour des métropoles. Les espaces ouverts du périurbain ne sont alors plus considérés comme des vides en attente d'urbanisation mais comme éléments structurants, faisant écho aux préoccupations des documents de planification dans le cas français où le paysage passe du décor au territoire (Moquay et al., 2007). 
3 Cette contribution questionne le rôle du paysage dans les politiques de planification pour repenser l'articulation entre différentes échelles de décision, et s'inscrit dans le renouveau à la fois de la recherche sur le paysage et les espaces ouverts, et de celle sur le devenir des espaces périurbains. Il s'agit ici de compléter un ensemble de travaux qui ont pu se concentrer sur des types d'espaces à enjeux paysagers comme les franges urbaines (Douence et Laplace-Treyture, 2016), les interfaces entre espaces bâtis et espaces non bâtis, supports de nouvelles pratiques pour les ménages dans le périurbain des métropoles (Bonin et al., 2016 ; Buyck, 2010 ; Germaine et al., 2017 ; Poulot, 2013). En écho, la planification s'est approprié le paysage via une "inversion du regard" (Davodeau, 2005), où le paysage devient outil d'aménagement mais aussi de construction politique nécessitant une approche négociée autour des représentations des différents acteurs.

4 Les résultats présentés ici sont issus d'un programme de recherche-action Interreg Alpine Space, LOS_DAMA! (Landscape and Open Space Development in Alpine Metropolitan

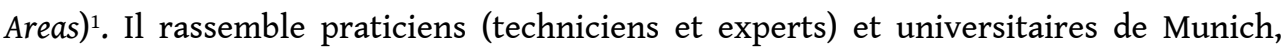
Vienne, Salzbourg, Trente, la région Piémont, Ljubljana et Grenoble, pour mettre en place des activités et outils au service de la valorisation des paysages périurbains de ces métropoles alpines. À ce volet technique répond une ambition politique, via l'appartenance à la Stratégie de l'Union européenne pour la région alpine (SUERA), visant à influencer la fabrique des politiques européennes et y affirmer l'importance d'une approche paysagère dans la planification et le financement de projets par les fonds de cohésion.

5 La méthodologie adoptée dans cet article repose sur une analyse de contenu, y compris lexicométrique, des Pilot action plans, documents stratégiques coconstruits dans le cadre du projet par les collectivités impliquées et les partenaires scientifiques ${ }^{2}$, et d'entretiens semi-directifs menés avec ces praticiens. Cette approche permet d'élaborer un diagnostic des politiques publiques de paysage et des thèmes connexes, et de la façon dont ces acteurs les présentent sur une scène de mise en discussion européenne.

6 Ainsi, dans quelle mesure la coopération de nature réticulaire entre les partenaires du projet LOS_DAMA!, au niveau transnational, s'articule-t-elle avec la production de politiques publiques de paysage "situées" dans un territoire donné et un contexte administratif et juridique déterminé ? La participation à ce réseau permet-elle aux divers acteurs engagés de produire des référentiels pour les politiques de paysage, ou du moins des modes d'agir sur le paysage, convergents?

7 Après avoir présenté le contexte de production des politiques et actions du projet, nous établirons un diagnostic des configurations d'action publique sur le paysage et des biais dans les politiques publiques ou les héritages de planification à résoudre sur les terrains évoqués. Enfin, nous proposerons des éléments de convergence sur l'appréhension de ces paysages par l'ancrage territorial des actions déployées (selon les échelles et les outils développés).

\section{Relire les paysages périurbains dans un contexte multiniveau et partenarial : le prisme de LOS_DAMA !}

8 Via la politique de cohésion et les programmes de coopération de type Interreg, on assiste à une européanisation de la planification spatiale (Colomb, 2007) qui se 
caractérise par une importance accordée aux processus de monitoring des changements apportés par les projets et aux dynamiques d'apprentissage. Ici, hormis le rôle de l'UE dans la reconnaissance des paysages ordinaires par la Convention européenne du paysage (Sgard et al., 2010), il s'agit de saisir l'européanisation des politiques publiques de paysage par la circulation de bonnes pratiques qui sous-tendent ces processus.

\section{Vers une vision européenne du paysage dans l'action publique?}

9 L'Union européenne, qui impulse des programmes de coopération thématiques interrégionaux, agit comme médiatrice du transfert de politiques ou de modes de faire entre cadres nationaux et acteurs représentant différentes échelles territoriales (Colomb, 2007). Ces programmes véhiculent également une certaine vision du paysage, par les partenaires mobilisés et les modèles sollicités.

Par ailleurs, les dynamiques périurbaines deviennent une préoccupation européenne en raison notamment du manque d'harmonisation statistique les concernant et de leurs conséquences sur de nombreux secteurs tels que le paysage, la biodiversité et la santé. Dans un contexte de croissance périurbaine soutenue, le programme Plurel (2007-2011) s'est ainsi penché sur les relations urbain-rural en mettant à disposition des acteurs un diagnostic pour initier une démarche comparative à l'échelle européenne (Chéry, 2010). D'autres programmes se sont attachés à traiter le paysage davantage sous l'angle des services écosystémiques et des solutions basées sur la nature dans les espaces métropolitains, selon un regard écologiste, comme Green Surge (2013-2017) ou Green Keys (2005-2008) (Buijs et al., 2015 ; Hansen et al., 2016, 2017). Ce premier programme a permis de diffuser le concept d'infrastructure verte urbaine auprès des décideurs locaux des villes partenaires, véhiculant ainsi une vision du paysage centrée sur l'interconnexion des espaces verts (Hansen et Rall, 2014), mais questionnant peu le paysage comme médium entre environnement et sociétés.

Sept terrains d'expérimentation, sept visions du périurbain dans les Alpes 
Figure 1. Carte de localisation des sept régions métropolitaines impliquées dans LOS_DAMA !

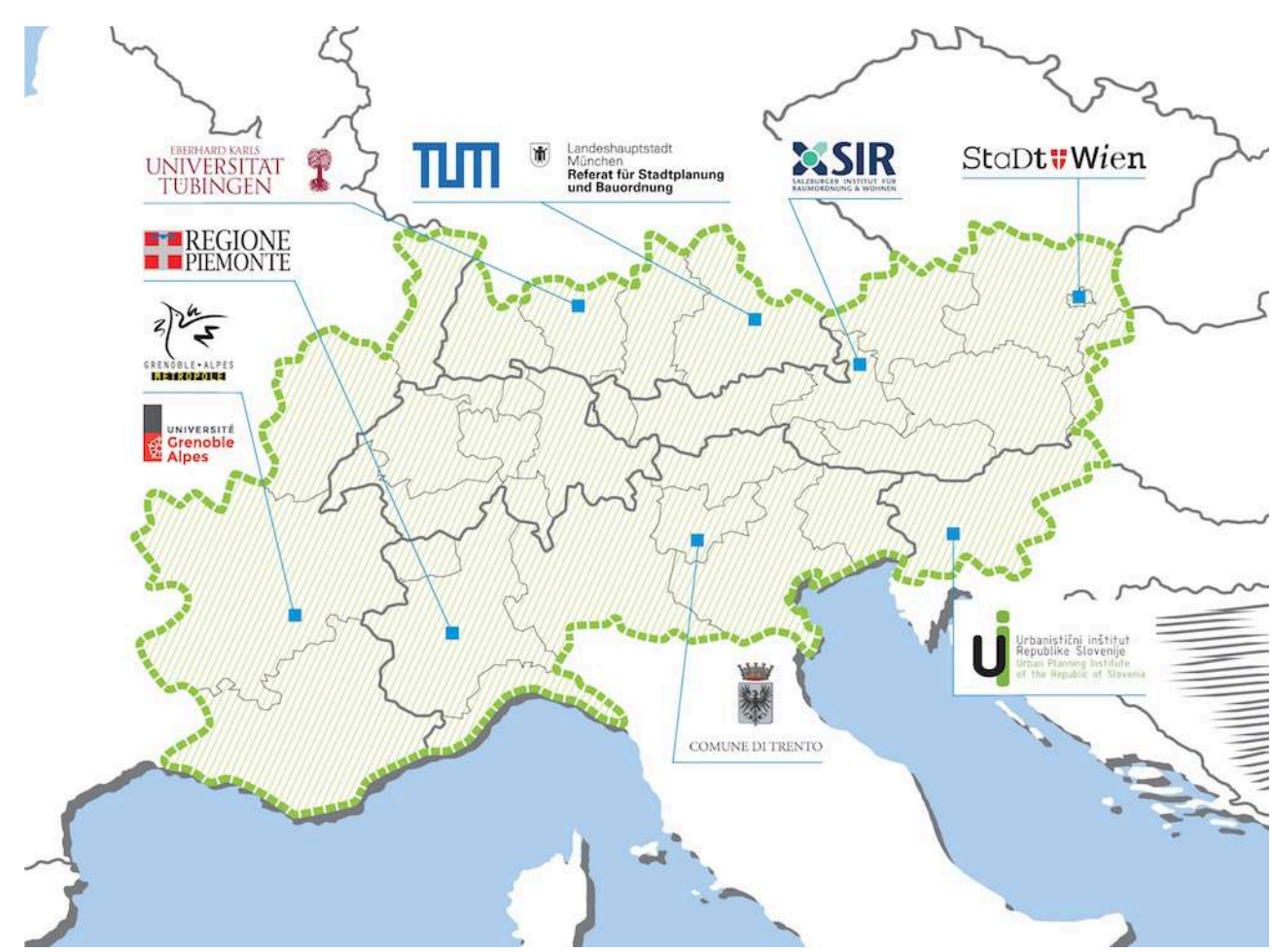

11 Les sept régions métropolitaines étudiées dans le projet révèlent autant de visions du périurbain, même si le rôle des contextes nationaux (systèmes de planification spatiale) demeure prégnant, notamment pour les cas italien et autrichien. Ces régions véhiculent à la fois les différentes représentations du périurbain (selon le zonage utilisé, le périmètre de la zone urbaine fonctionnelle, le lien à la ville-centre par les navetteurs), et ses différentes missions dans sa position d'interface (assimilé à l'étalement urbain ou bien vecteur de nouvelles relations ville-campagne) et différents usages (prédominance d'usages productifs, récréatifs, ou au contraire préservation de la nature). En outre, elles portent également des configurations paysagères différenciées, spécifiques aux lieux au gré de l'influence alpine, du rôle de la pente et des écosystèmes locaux, bien qu'on puisse observer des tendances similaires en termes de zones humides ou d'évolution des prairies. Cependant, la dimension alpine reste peu questionnée dans le programme, étant davantage assimilée à un cadre donné, à un décor, sans être considérée dans son rôle structurant, en créant un périurbain particulier car influencé par la pente.

12 La vision du périurbain qui transparaît dans les Pilot action plans demeure assez binaire avec une domination du diptyque ville/campagne. Ainsi, Vienne et Ljubljana sont confrontées toutes deux à une croissance démographique importante et à un développement périurbain assimilé à l'étalement que les espaces naturels, agricoles et forestiers doivent réguler. Toutefois, Trente et Grenoble, qui se rapprochent par leurs caractéristiques de site, insistent sur l'accès aux espaces périurbains, qu'il s'agisse de connectivité physique ou écologique. Une approche moins spatialisée permet de détacher le cas de la région Piémont où le périurbain et la périurbanisation sont passés sous silence pour privilégier un regard écologique par les solutions basées sur la nature. 
13 Les données Corine Land Cover fournissent une approche diachronique permettant de retracer les dynamiques paysagères périurbaines via l'évolution de la couverture du sol; elles fournissent des éléments sur les formes de la périurbanisation dans les terrains d'étude. À ce titre, entre 1990 et 2012, tandis que les zones urbanisées affichent une forte progression (plus de $12 \%$ en moyenne), notamment à Grenoble et à Salzbourg (respectivement $+26 \%$ et $+19 \%$, imputable au caractère enclavé des deux sites), les zones agricoles sont en net recul, et plus particulièrement les prairies (en recul de près de $10 \%$ en moyenne) et les zones agricoles hétérogènes (- $8 \%$ en moyenne). En agrégeant ces données pour obtenir une distribution selon les zones artificialisées et celles qui ne le sont pas, les sites de Munich, Turin et Grenoble apparaissent particulièrement touchés par un recul des surfaces naturelles agricoles. Les cartes réalisées démontrent des tendances spatiales communes mais aussi certains effets de site. Ainsi, Grenoble et Trente, deux métropoles alpines encaissées, présentent une périurbanisation qui se conforte sur les contreforts des massifs; cela est particulièrement visible à Grenoble avec une artificialisation des sols à l'est de l'agglomération, sur les balcons de Belledonne au-dessus de la vallée du Grésivaudan au nord-est du cœur urbanisé, ainsi que dans le Sud grenoblois, au-delà de la frange verte, vers l'Oisans, dans un contexte de déprise agricole. D’autres terrains présentent une périurbanisation « en doigts de gants » sous la forme d'un développement radial tel que Ljubljana (où ce phénomène est récent en raison de la transition postsocialiste et de la privatisation du stock de logements) ou la ville de Salzbourg (sous forme de villagesrues avec des lotissements en raquette), plus particulièrement confrontée à la rareté d'un foncier abordable. Enfin, Turin et Vienne présentent des formes mixtes de périurbanisation avec à la fois un développement radial mais aussi une densification en lisière urbaine pour Turin, et autour de noyaux villageois dans les Länder ruraux environnants de Basse-Autriche et du Burgenland pour la capitale autrichienne qui fait face à une croissance démographique soutenue (elle devrait atteindre le seuil des deux millions d'habitants d'ici 2026).

Tableau 1. Évolution des surfaces selon la base de données Corine Land Cover entre 1990 et 2012 , en $\%$

\begin{tabular}{|c|c|c|c|c|c|c|c|}
\hline & Grenoble & Ljubljana & Trento & Turin & Salzbourg & Vienne & Munich \\
\hline Zones urbanisées & 26,1 & 0,5 & 12,0 & 10,8 & 19,1 & 9,4 & 8,0 \\
\hline Zones industrielles ou commerciales & & & & & & & \\
\hline et réseaux de communication & 19,9 & 11,7 & 17,0 & 43,2 & 399,5 & 75,3 & 44,6 \\
\hline Mines, décharges et chantiers & 8,0 & 1,9 & 5,5 & 110,5 & 20,1 & 52,2 & $-20,7$ \\
\hline Espaces verts artificialisés, non & & & & & & & \\
\hline agricoles & 5,4 & 102,6 & 0,0 & 24,2 & 0,0 & 110,9 & 58,1 \\
\hline Terres arables & 17,7 & $-0,2$ & 0,0 & $-3,2$ & 753,4 & $-3,1$ & 13,1 \\
\hline Cultures permanentes & $-2,4$ & 0,0 & 29,1 & $-2,6$ & 0,0 & $-4,2$ & $-30,6$ \\
\hline Prairies & 0,7 & $-1,1$ & $-37,8$ & $-24,7$ & $-17,3$ & $-45,5$ & 57,8 \\
\hline Zones agricoles hétérogènes & $-16,9$ & 0,4 & $-16,3$ & $-2,2$ & 69,7 & 3,7 & $-94,2$ \\
\hline Forêts & $-0,5$ & $-0,3$ & 0,9 & $-6,5$ & $-1,9$ & 1,0 & 2,4 \\
\hline Milieux à végétation arbustive et/ou & & & & & & & \\
\hline herbacée & $-3,2$ & 2,2 & 6,3 & 27,9 & 10,9 & 84,8 & $-54,7$ \\
\hline $\begin{array}{l}\text { Espaces ouverts, sans ou avec peu de } \\
\text { végétation }\end{array}$ & 6,1 & $-0,1$ & 11,4 & $-28,8$ & $-4,5$ & $-10,2$ & 0,0 \\
\hline Zones humides intérieures & $-20,8$ & 5,0 & 0,0 & 0,0 & 11,7 & $-4,7$ & $-38,8$ \\
\hline Eaux continentales & $-12,9$ & $-12,7$ & 0,0 & $-16,2$ & 1,9 & 2,1 & 2,8 \\
\hline
\end{tabular}

Aurore Meyfroidt, 2018. 
Figure 2. Évolution de la répartition des terres entre surfaces artificialisées et surfaces naturelles, selon la base de données Corine Land Cover (1990-2012)

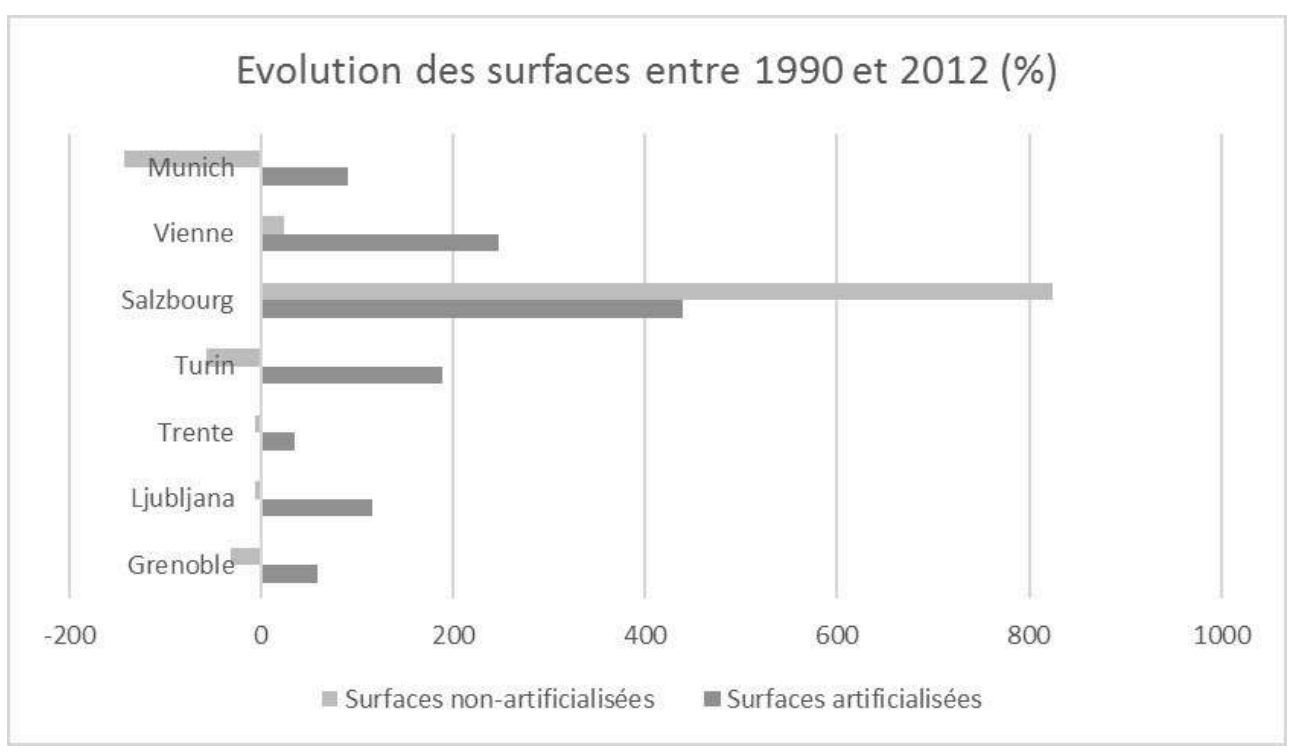

Aurore Meyfroidt, 2018.

Figure 3a. Évolution de la couverture du sol pour les métropoles partenaires du projet : Grenoble

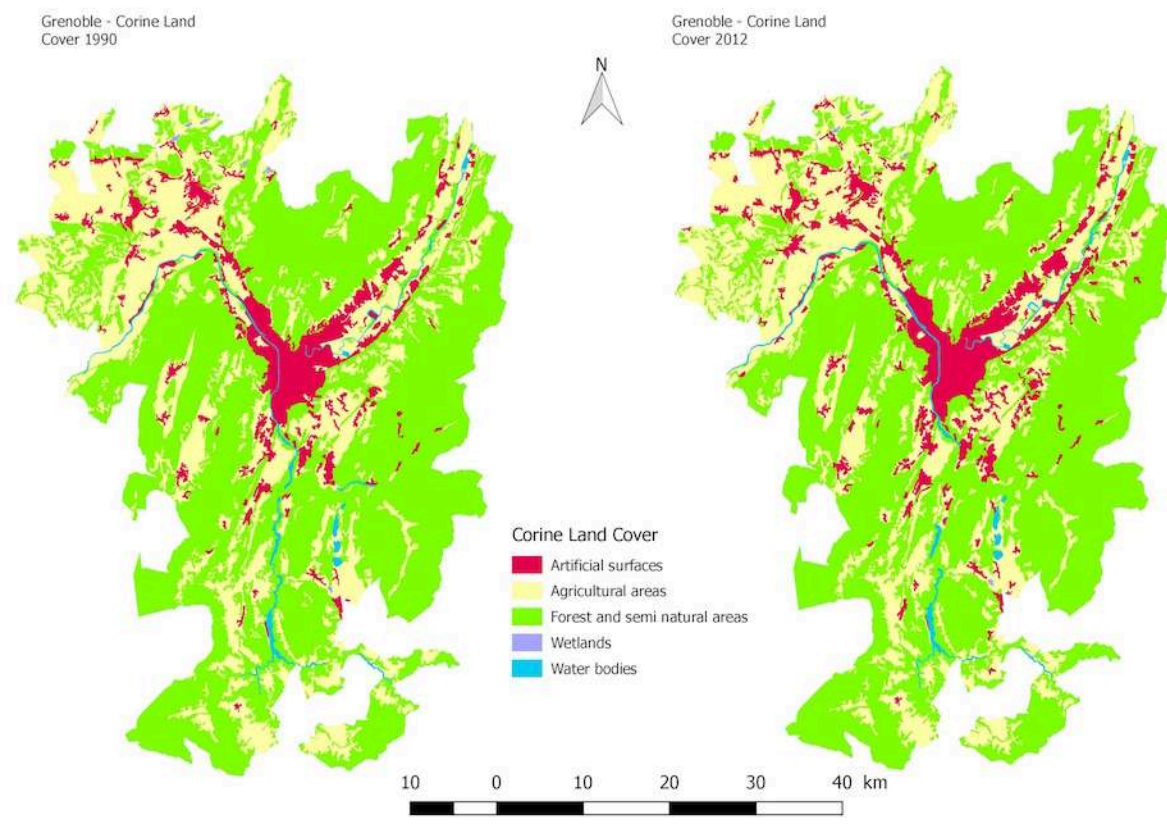

Source : Corine Land Cover ; réalisation : Céline Antunes, Grenoble Alpes Métropole. 
Figure 3b. Évolution de la couverture du sol pour les métropoles partenaires du projet : Ljubjana

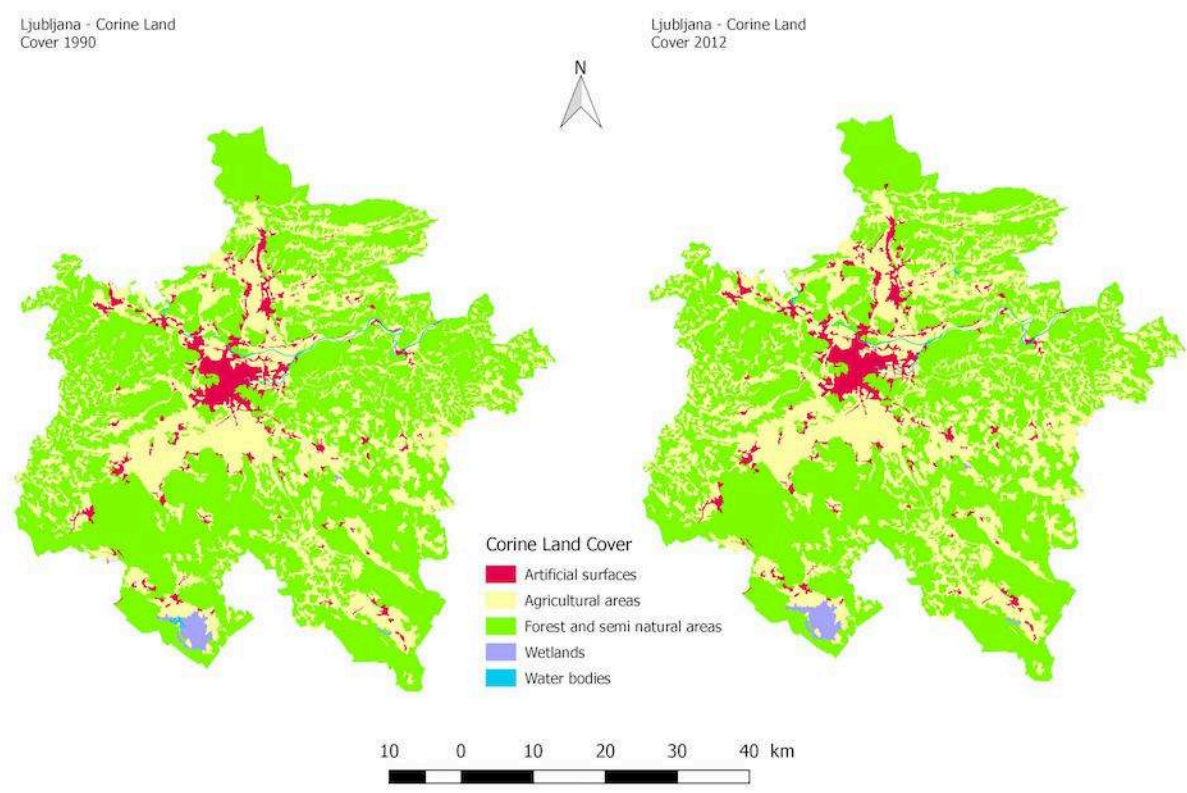

Source : Corine Land Cover ; réalisation : Céline Antunes, Grenoble Alpes Métropole.

Figure 3c. Évolution de la couverture du sol pour les métropoles partenaires du projet : Munich

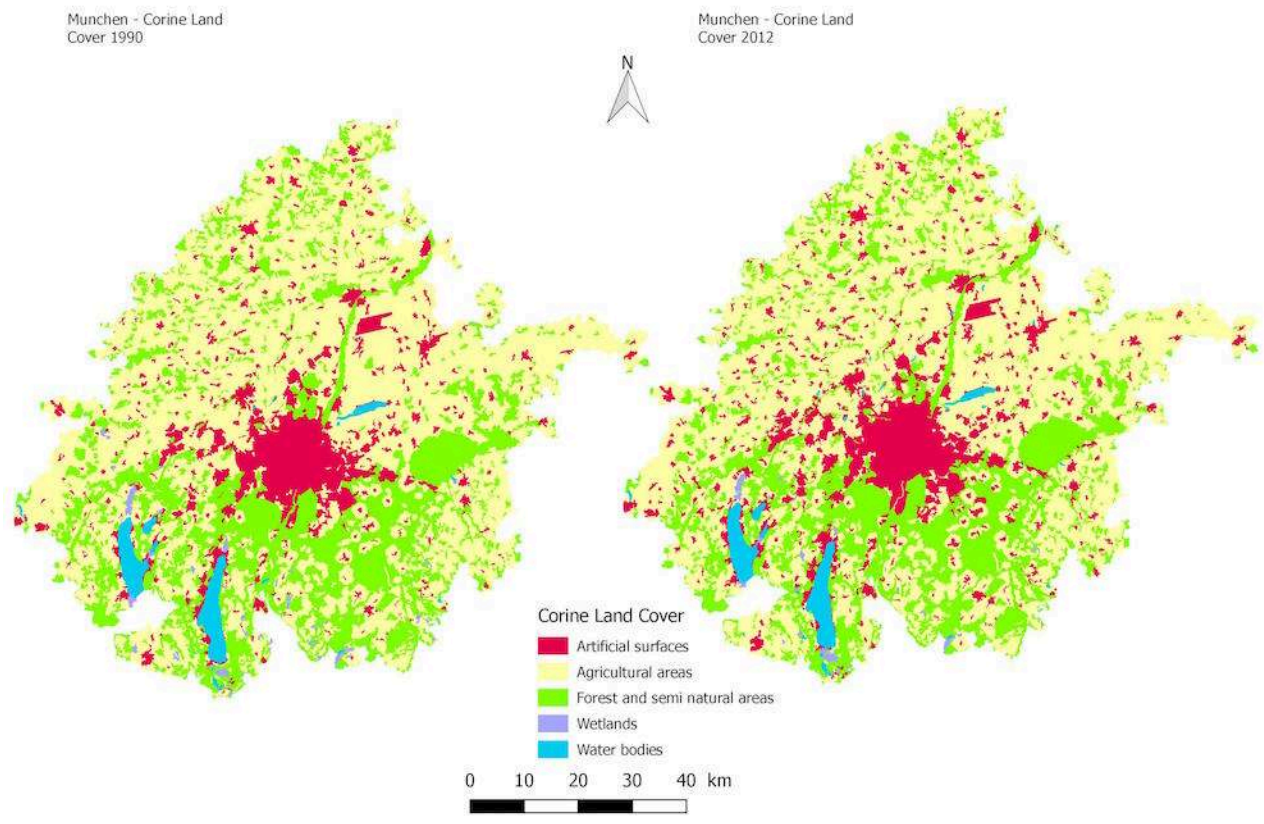

Source : Corine Land Cover ; réalisation : Céline Antunes, Grenoble Alpes Métropole. 
Figure 3d. Évolution de la couverture du sol pour les métropoles partenaires du projet : Salzbourg

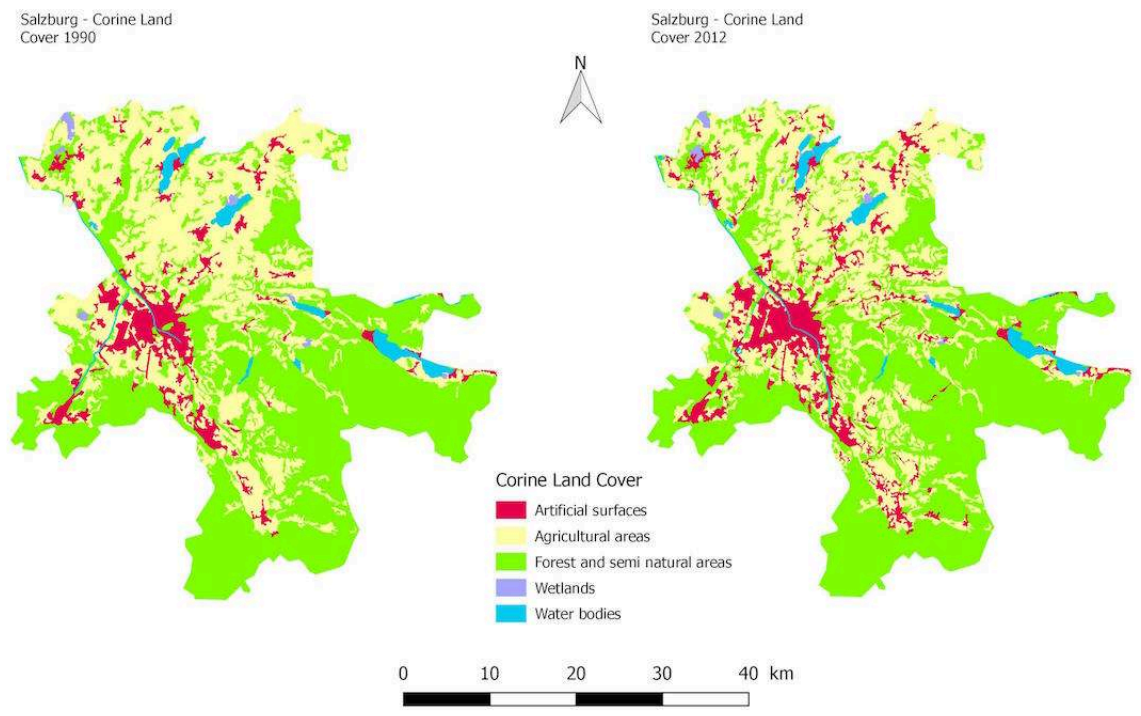

Source : Corine Land Cover ; réalisation : Céline Antunes, Grenoble Alpes Métropole.

Figure 3e. Évolution de la couverture du sol pour les métropoles partenaires du projet : Turin

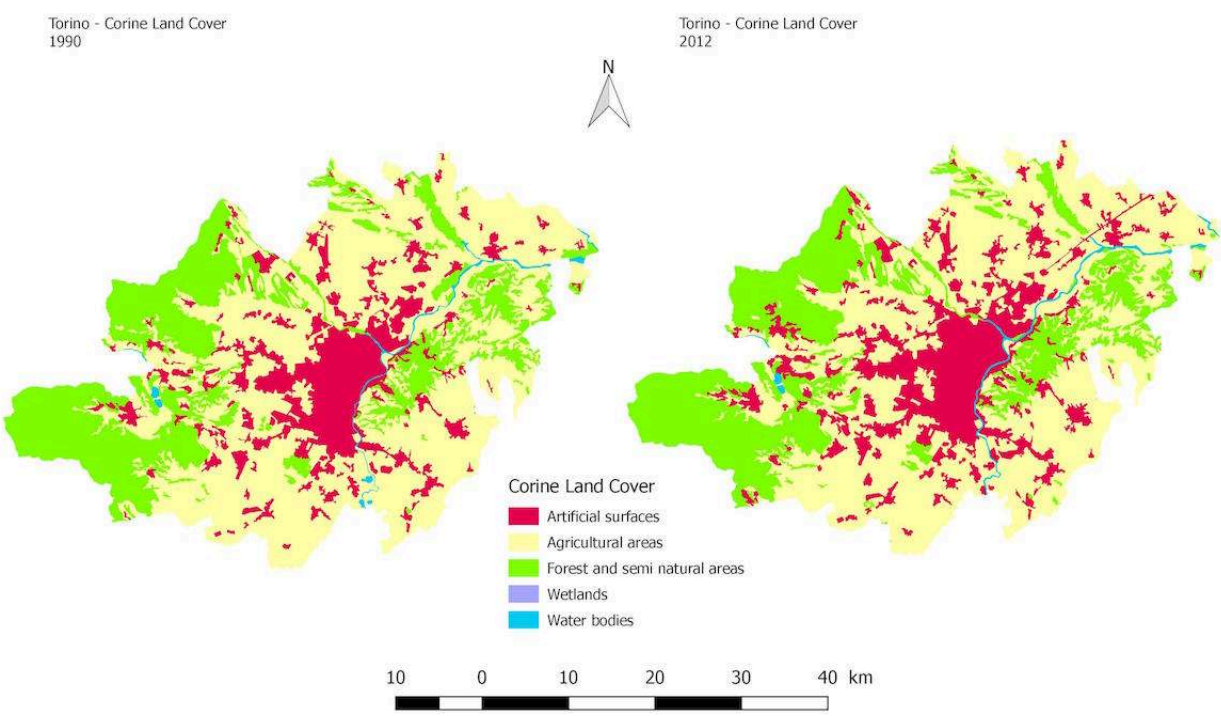

Source : Corine Land Cover ; réalisation : Céline Antunes, Grenoble Alpes Métropole. 
Figure 3f. Évolution de la couverture du sol pour les métropoles partenaires du projet : Trente

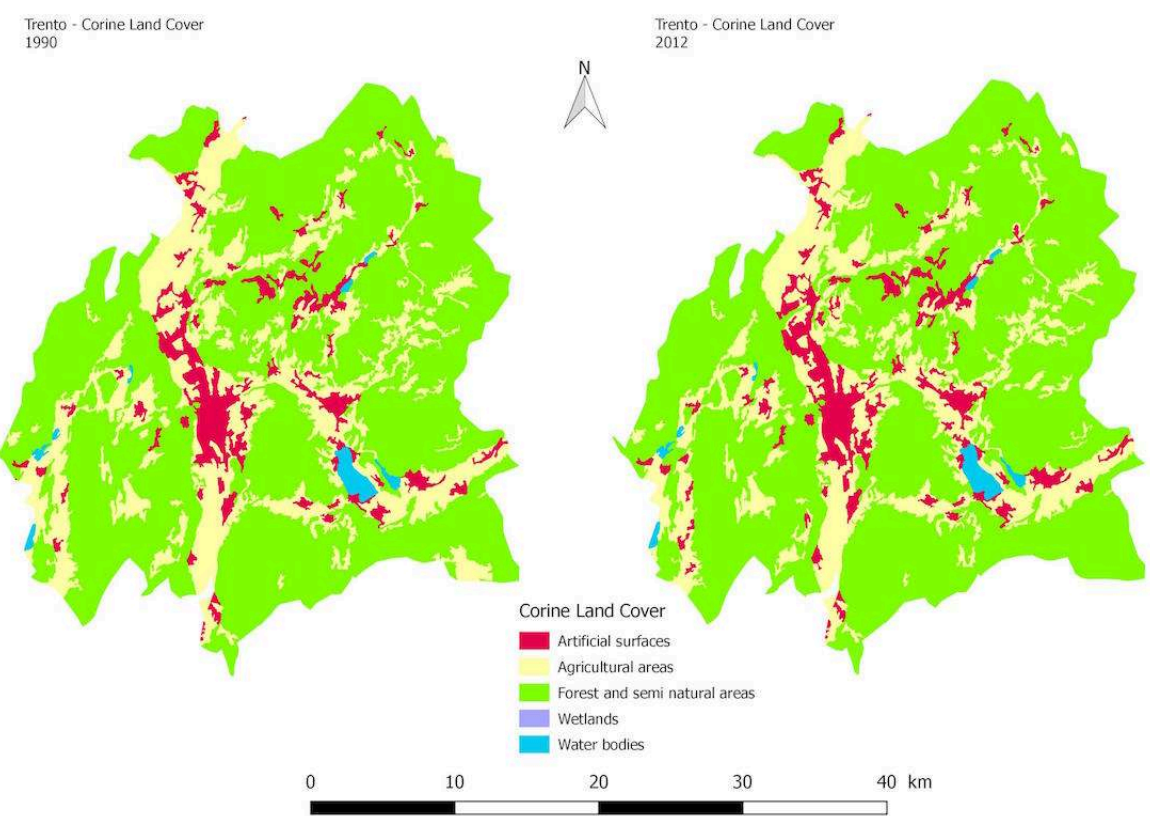

Source : Corine Land Cover ; réalisation : Céline Antunes, Grenoble Alpes Métropole.

Figure 3g. Évolution de la couverture du sol pour les métropoles partenaires du projet : Vienne

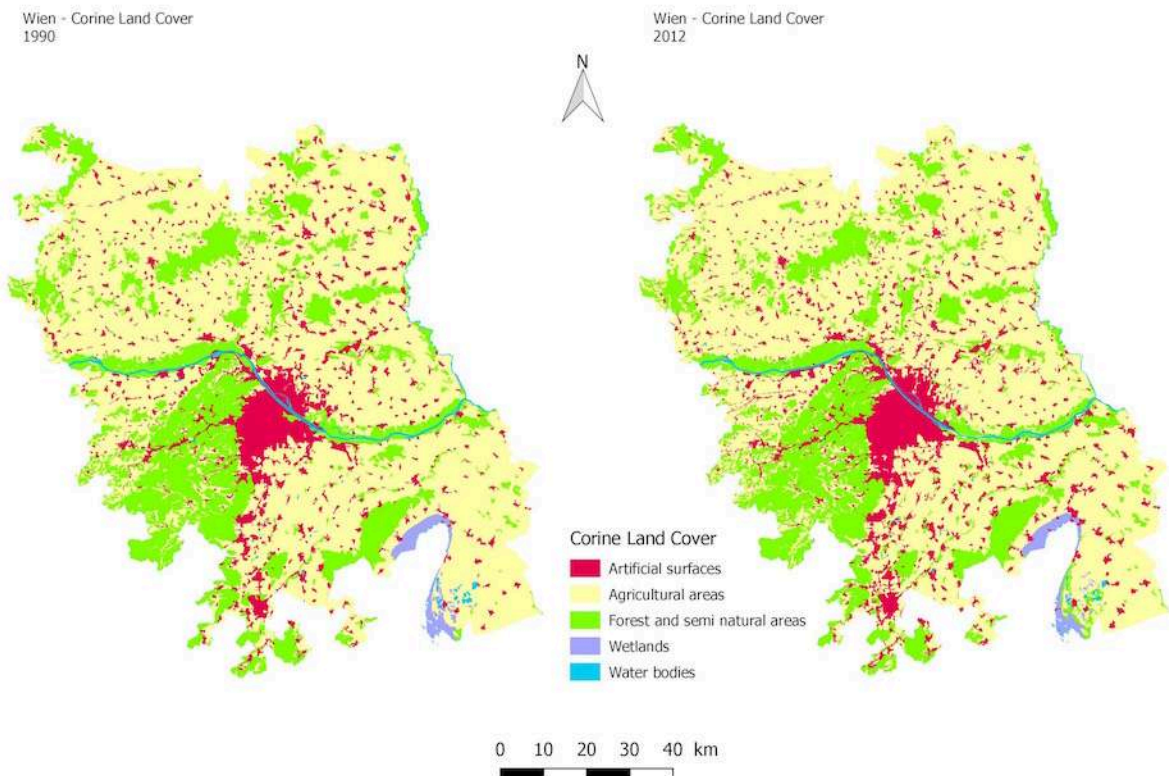

Source : Corine Land Cover ; réalisation : Céline Antunes, Grenoble Alpes Métropole.

14 Hormis les données Corine Land Cover, l'Agence européenne pour l'environnement développe des indicateurs sur les espaces verts urbains ${ }^{3}$ et plus particulièrement celui de Effective Green infrastructure ${ }^{4}$ qui reflète la répartition des espaces naturels, agricoles 
et forestiers dans les zones de contact ville/campagne des métropoles. À ce titre, on peut observer des hiatus entre la répartition des espaces verts à l'échelle de la métropole et ce dernier indicateur: tandis que Vienne présente $30 \%$ de son infrastructure verte en lisière ville/campagne (en raison d'une présence importante de parcs et de la forêt viennoise à l'intérieur du périmètre communal), on observe une surconcentration dans le cas de Salzbourg et Trente avec les héritages des ceintures vertes.

Tableau 2. Indicateurs d'infrastructures vertes en 2012.

\begin{tabular}{|l|l|l|l|}
\hline & \multicolumn{3}{|l|}{ Urban Green Infrastructure Indicators (EEA) } \\
\hline Name & $\begin{array}{l}\text { Share of green } \\
\text { urban areas }\end{array}$ & $\begin{array}{l}\text { Distribution of green } \\
\text { urban areas }\end{array}$ & $\begin{array}{l}\text { Effective green } \\
\text { infrastructure }\end{array}$ \\
\hline Grenoble & $60,9 \%$ & $17,5 \mathrm{~m} / \mathrm{ha}$ & $72,4 \%$ \\
\hline Ljubljana & $72,5 \%$ & $26,4 \mathrm{~m} / \mathrm{ha}$ & $77,7 \%$ \\
\hline Munich & $35,8 \%$ & $24,8 \mathrm{~m} / \mathrm{ha}$ & $44,5 \%$ \\
\hline $\begin{array}{l}\text { Piedmont } \\
\text { (Torino) }\end{array}$ & $35,9 \%$ & $34,1 \mathrm{~m} / \mathrm{ha}$ & $47,7 \%$ \\
\hline Salzburg & $50,3 \%$ & $35,5 \mathrm{~m} / \mathrm{ha}$ & $82,5 \%$ \\
\hline Trento & $80,4 \%$ & $21,3 \mathrm{~m} / \mathrm{ha}$ & $78,9 \%$ \\
\hline Vienna & $49,5 \%$ & $32,4 \mathrm{~m} / \mathrm{ha}$ & $30,1 \%$ \\
\hline
\end{tabular}

Source : Agence européenne pour l'environnement ; réalisation : Martina van Lierop, TUM.

15 Face à cette diversité de situations, le projet se veut un laboratoire d'expérimentation pour déployer des méthodes et des outils au service d'une action publique multiniveau et transversale.

\section{S'appuyer sur des documents stratégiques coproduits pour saisir la réflexivité des acteurs locaux sur l'objet " paysage périurbain »}

La valeur ajoutée du traitement des dynamiques des paysages périurbains dans un cadre européen réside dans la diversité des positionnements des acteurs, entre recherche et pratique et dans un contexte interculturel.

\section{Un document coconstruit : le Pilot Action Plan}

L'utilisation de documents visant à la fois la description des politiques publiques en place et la présentation des activités des collectivités partenaires est assez récurrente dans le cadre de projets européens. Par exemple, on mentionnera le projet Re-Seeties (Towards resource-efficient local communities in South East Europe) où les villes partenaires ont élaboré des Local Strategies and Action Plan avec des experts, en tant qu'outils de monitoring du projet, en termes de maturité des actions proposées (Di Leo et Salvia, 2017). Ce type de document renforce la construction de capacités et la création de réseaux en développant des outils d'aide à la décision transférables d'un terrain à l'autre. D'autres programmes européens, comme le programme Urbact, présentent aussi des expériences similaires de rédaction de plans d'action locaux (Brand, 2018) pour décentrer les regards situés de l'action publique et encourager la coopération audelà des secteurs. 

instruments de contenus ou de processus, et repose sur un modèle de document commun construit par les universités partenaires du projet et deux acteurs locaux. Son élaboration fait écho aux résultats d'autres programmes européens, notamment Green Surge, par la reprise des catégories d'analyse de multifonctionnalité et des services écosystémiques. Il présente les politiques publiques concernant le paysage et les secteurs connexes (biodiversité, eau, forêt...), les enjeux spécifiques au terrain, des exemples de réussite et d'échec, la présentation de valeur ajoutée du projet et surtout des activités pilotes qui sont en train d'être mises en place dans les sept métropoles. Les activités pilotes sont de deux types. Certaines sont d'ordre méthodologique, liées à des processus d'action publique annexes (exemple de la master class des décideurs locaux pour accompagner la mise en place d'une opération aménagement et programmation paysage à Grenoble) ou à des outils (outil de visualisation pour un projet mené à Munich). D'autres sont liées à un territoire, qu'il s'agisse du périurbain dans sa globalité (projet de compensation foncière à Salzbourg) ou sur un espace délimité (aménagement d'un espace naturel sur la frange verte de Vienne).

19 Ces sept plans d'action pilote (PAP) ne visent pas une comparaison terme à terme mais plutôt l'identification de traits communs dans ce qu'apporte l'implication des collectivités dans le projet européen, par la manière de concevoir le paysage et de mobiliser les acteurs.

\section{Un degré de maturité varié des politiques de paysage : diagnostic des biais et faiblesses}

La présentation des politiques publiques de paysage dans les plans d'action pilote sert à la fois de cadre de l'action mais aussi de prise de conscience des manques dans l'action publique sur le paysage. Ces plans renvoient à la répartition des politiques à différentes échelles, à l'absence de synergies entre acteurs ou encore à des approches du paysage centrées uniquement sur la préservation de l'environnement, contrairement à une approche holistique promue par la Convention européenne du paysage ${ }^{5}$.

21 Cette réflexion sur l'approche multiscalaire du paysage s'inscrit tout d'abord dans une nouvelle lecture de la gouvernance environnementale (Bulkeley, 2005) caractérisée par une hybridation entre échelles et une multiplicité d'acteurs impliqués. Ces dispositifs de la gouvernance environnementale entrent en écho avec les dimensions spatiales des politiques publiques, au sein d'une perspective multiniveau, où les échelles des politiques, considérées comme constructions sociales, ne pourraient répondre qu'imparfaitement aux déterminants matériels et naturels des phénomènes environnementaux (Haase et al., 2014) : le paysage intervient alors comme médiateur entre ces deux pôles, mais sa gouvernance doit rester suffisamment dynamique pour surpasser le localisme (Görg, 2007).

Dans les plans d'action pilote, les partenaires du projet présentent une répartition variée des compétences liées au paysage selon les échelles. Les échelles régionales et locales sont privilégiées, notamment dans le cas de Munich, Salzbourg, et Vienne, en raison du cadre national fédéraliste. Quant à l'échelle nationale, elle est davantage présente dans les politiques annexes (agriculture, biodiversité et forêt), tandis que les politiques de projet urbain et de planification privilégient le local. Hormis l'exemple fédéraliste, d'autres spécificités émergent comme le lien fort à la planification

Projets de paysage, $19 \mid 2018$ 
régionale dans le cas italien, ou encore la relative «jeunesse» des documents en Slovénie, en raison de la transition postsocialiste. Certaines échelles paraissent passées sous silence, notamment l'échelle infralocale, en raison du profil des partenaires engagés, et l'échelle intercommunale. Les compétences à cette dernière échelle semblent être limitées à l'exemple grenoblois, où les intercommunalités, suite aux réformes territoriales successives, sont le principal levier d'implémentation des politiques de paysage et de biodiversité. Ce niveau n'est cependant pas absent sur les autres terrains mais il reste cantonné au domaine du projet, sans compétences propres. En lien avec cette répartition par échelles, les documents nous éclairent sur les conceptions du paysage portées par ces textes, d'une approche holistique à une fragmentation sectorielle. Ainsi, l'approche écologiste, centrée sur la conservation de la nature, distingue à nouveau les terrains germaniques, tandis que les autres collectivités adopteraient une vision plus holistique du paysage, avec certaines nuances : la Slovénie envisage le paysage au prisme du développement durable, la France opère un glissement assez récent vers cette vision holistique, et enfin les deux sites italiens dénotent un rôle moteur du paysage dans la planification.

Tableau $3^{6}$. Répartition des politiques de paysage selon les échelles et les approches du paysage d'après les plans d'action pilote réalisés durant le projet

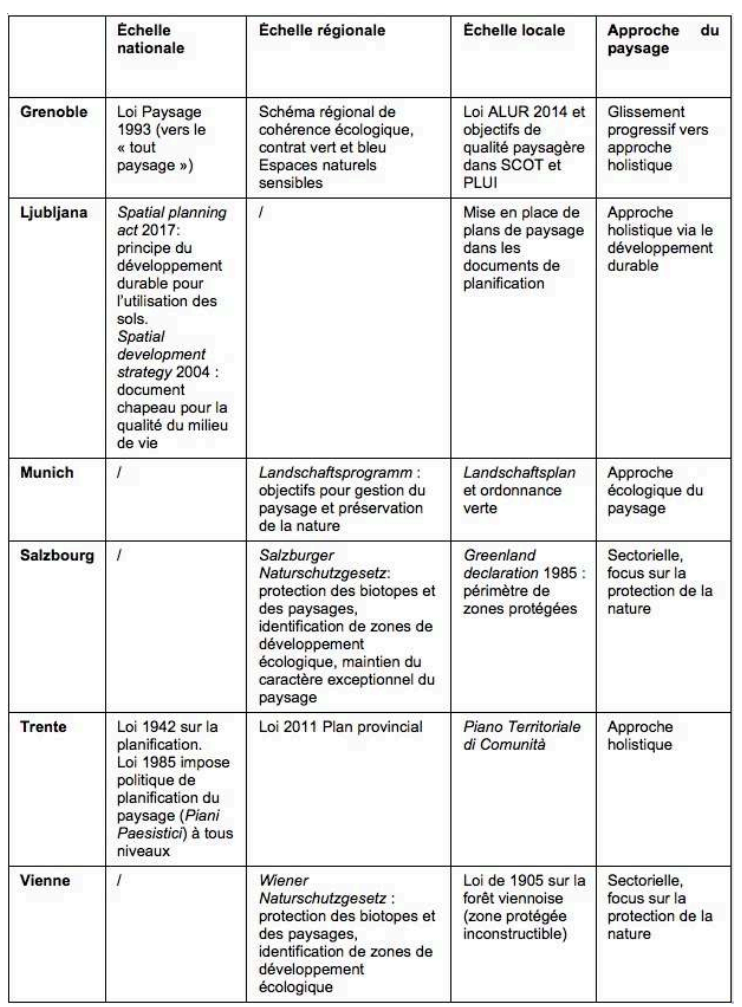

Réalisation : Aurore Meyfroidt, 2018.

Une visualisation des termes utilisés dans le discours des experts qui ont rédigé ces plans d'action locale confirme le pôle écologiste de ce diagnostic, apporte également des éclaircissements sur le lien au terrain et les missions attribuées au paysage dans l'action publique. En rassemblant les descriptions des politiques publiques dans un même corpus, en différenciant selon le terrain concerné, l'analyse lexicométrique ${ }^{7}$, via les occurrences des termes et leur proximité lexicale, révèle à la fois les thématiques et 
enjeux associés aux politiques de paysage et connexes, et les facteurs différenciant les collectivités.

Figure 4. Analyse lexicométrique de la description des politiques publiques

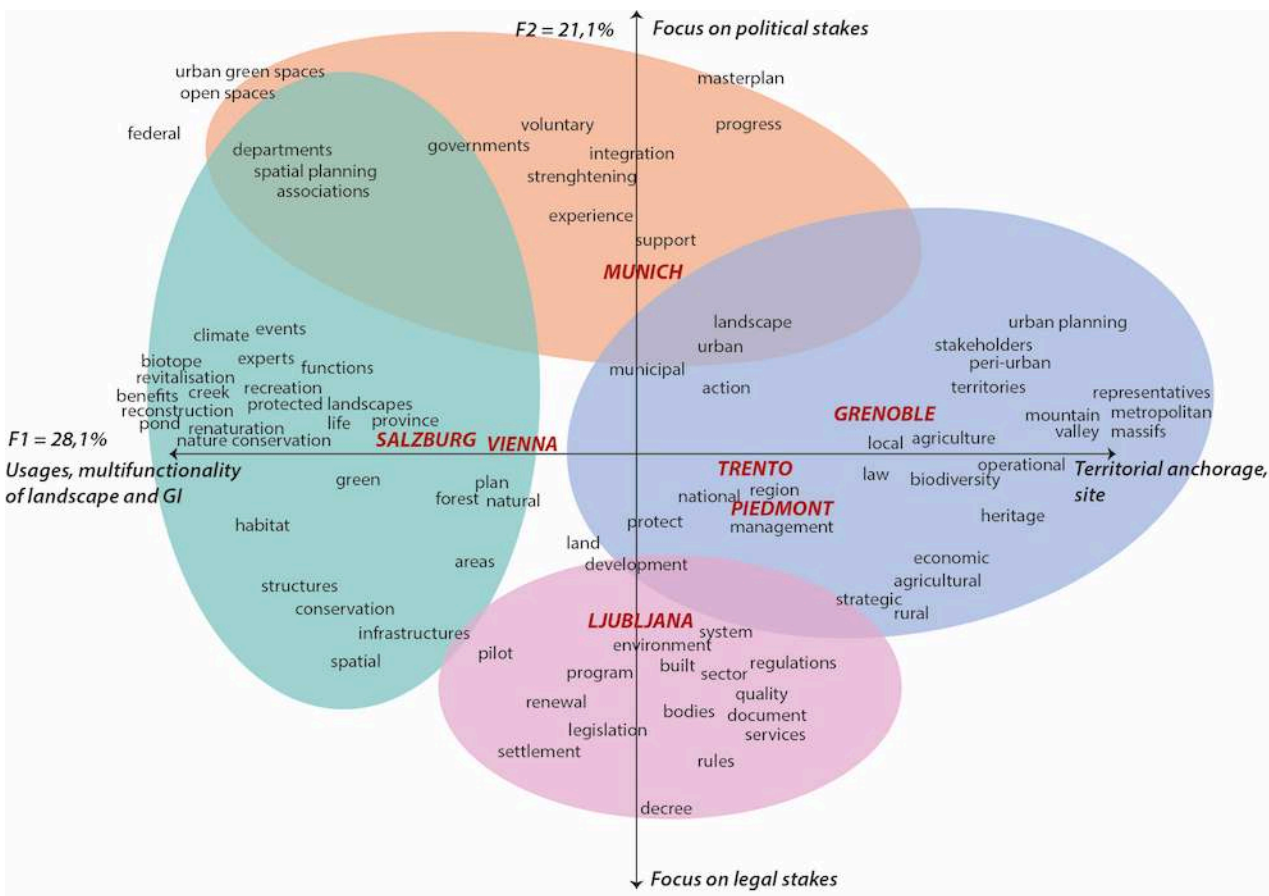

Source : Plans d'action pilote ; réalisation : Aurore Meyfroidt, 2018. estimons ce taux satisfaisant en raison de la petite taille du corpus et de l'inégale qualité des réponses aux questions fournies par les collectivités. Le premier (F1) distingue une approche du paysage (par les fonctions et usages) basée sur la protection de la nature ("nature conservation», "renaturation», "biotope») d'une orientation beaucoup plus ancrée dans les territoires, où le paysage serait porteur d'un projet de développement territorial («territories», "stakeholders», "urban planning», " metropolitan»). Le paysage est alors considéré via son volet environnemental, voire écologique, surtout par les villes de Salzbourg et de Vienne, tandis qu'il est perçu comme outil au service d'un développement spatial multiacteur par Grenoble et, dans une moindre mesure, par les villes italiennes. Quant au deuxième axe (F2), il reflète le processus d'action publique à l'œuvre, du réglementaire ("decree», "rules», " legislation») au projet politique ("experience», "voluntary»), opposant une vision slovène où l'approche paysage du programme s'inscrit dans une lignée de documents institutionnels, à un projet munichois où il permet de promouvoir une démarche de coconstruction d'un projet politique.

Les collectivités se saisissent des leviers offerts par ce projet européen de façon assez différenciée, l'ambition de ce dernier étant de donner des outils pour transcender les arènes sectorielles et les échelles. Les éléments de différenciation entre collectivités selon l'état des lieux des politiques publiques se doivent d'être confrontés aux actions projetées par chaque partenaire dans le cadre du projet, révélant des priorités distinctes. 


\section{Résultats : les trois unités révélées des paysages périurbains}

26 Les activités pilotes présentées par chaque collectivité partenaire dans son plan d'action révèlent des éléments de convergence entre ces acteurs, en termes d'enjeux ou de méthodes, et proposent des solutions expérimentales pour remédier au manque de synergie dans la gouvernance du paysage, horizontale (entre types d'acteurs) ou verticale (entre échelles). Plus largement, ces activités questionnent les conditions de production des politiques publiques de paysage en local, dans le rapport au lieu, à la temporalité et aux acteurs.

\section{Unité de lieu : la « mise en territoire » du paysage pour connecter espaces urbains et ruraux/montagnards}

Cette première unité envisage l'inscription spatiale des espaces ouverts dans les zones périurbaines et leur rôle hybride entre ville et campagne, telle que décrite dans les plans d'action pilote. L'analyse lexicométrique précédente a révélé une grande variation de la spécificité accordée au site et à l'ancrage territorial de ces espaces naturels, agricoles et forestiers, ce qui peut s'expliquer par le caractère assez désincarné, voire "aspatial», de la catégorie «infrastructure verte», utilisée principalement pour encourager la prise de conscience des décideurs politiques et développer des synergies entre acteurs (Albert et Von Haaren, 2014; Thomas et Littlewood, 2010). Plus globalement il s'agit d'observer quels projets de territoire à l'interface entre urbain et rural sont portés, via le discours sur le paysage et les espaces ouverts, tout en observant l'épaisseur de l'espace périurbain et son autonomie.

Cette inscription locale dépend tout d'abord des fonctions portées par le paysage dans ces espaces spécifiques. Ainsi, certains usages tels que l'agriculture porteraient davantage le ferment d'un lien retrouvé au territoire que la fonction de préservation de la nature. Ainsi, les acteurs de Trente utilisent l'agriculture comme levier dans les espaces périurbains :

«De plus, il y a un manque de coordination dans les politiques locales de biodiversité, d'agriculture et d'infrastructures vertes. Les activités pilotes de Trente se concentrent sur les stratégies et politiques pour améliorer la biodiversité et une agriculture durable en mobilisant à la fois les terrains publics et privés.» (PAP Trente, p. 7.)

Les espaces naturels, agricoles et forestiers révèlent-ils des rapports ville/campagne réinventés au service de l'autonomie du périurbain? Les motifs spatiaux des activités pilotes du projet révèlent trois configurations de ce lien : la frontière, l'interface et le réseau. La première figure renvoie à la fonction des espaces naturels, agricoles et forestiers comme barrière à l'urbanisation. Comme indiqué par Monique Toublanc et Sophie Bonin (2012), la trame verte et bleue peut être envisagée comme un «filet vert paysager pour endiguer l'étalement urbain » (p.4), dans une approche binaire entre espace à développer et espace naturel, ce dernier devant être aménagé ou promis à un aménagement futur. Il s'agit ici par exemple de maîtriser les formes de l'étalement urbain pour limiter le mitage en encourageant la place de l'agriculture dans l'aménagement périurbain; l'un des objectifs de la région Piémont dans le projet 
renvoie ainsi à : «[...] la maitrise de la dispersion du peuplement et la protection et l'amélioration de la nature avec des incitations pour une utilisation plus organisée et connectée à des politiques pour valoriser l'agriculture sous des formes durables pour les municipalités. » (PAP Piémont, p. 17.)

Cette fonction de barrière reflète davantage le besoin de mettre des limites à l'urbanisation du point de vue morphologique plus qu'institutionnel ou fonctionnel, pour atteindre un développement régional plus soutenable, comme en atteste l'exemple viennois : «Les paysages, les réseaux d'espaces verts et naturels, comme la Lobau, la forêt viennoise ou la Marche ne s'arrêtent pas aux frontières administratives. La région métropolitaine est une réalité - du point de vue physique, morphologique et fonctionnel. » (PAP Vienne, p. 34.)

31 Une deuxième figure, celle de l'interface, transcrit le caractère hybride des relations ville-campagne/montagne, au service d'une autonomie du périurbain. Les espaces ouverts participent de la construction territoriale et de la mise en valeur de l'hybridité pour fabriquer la ville-campagne (Poulot et al. 2016). Cette approche se manifeste dans les plans d'action pilote par une recherche du compromis entre usagers urbains et habitants périurbains, et une recherche de couture ente ville et campagne depuis un périurbain revalorisé. Ainsi, la ville de Munich insiste sur son rôle de «réservoir » de visiteurs pour les zones périurbaines en raison de leurs aménités, apportant une forme de redistribution des revenus via l'attractivité du paysage, mais aussi des pressions supplémentaires et d'une menace perçue par les locaux, ce qui nécessite de penser une forme de réciprocité. De la même façon, le périurbain viennois se dote d'un paysage à l'échelle régionale, lui permettant de devenir démonstrateur d'une intégration régionale : «Le paysage est déjà là, ancré, mais il faut le rendre visible, montrer que depuis ce paysage, on peut faire l'expérience de la ville. Qu'on peut avoir une relation à la Skyline de Vienne et aux Alpes depuis ces espaces-là. » Entretien avec les collectivités membres du projet, Vienne, 14 mars 2018.

32 Enfin, la figure du réseau renvoie à la mise en place d'une infrastructure verte multifonctionnelle qui devient support du développement urbain, questionnant cependant moins directement les catégories d'urbain, de périurbain et de rural: «L'espace du projet pilote est la Corona Verde (la couronne verte). La Corona Verde est un projet stratégique de la région Piémont qui a pour ambition de mettre en place une infrastructure verte en intégrant les résidences royales de la "Couronne des Trésors" (labellisées Unesco) qui entourent la ville de Turin en tant que ceinture verte. » «PAP Piémont, p. 27.)

\section{Unité de temps : la « mise en politique publique » du paysage}

33 La persistance d'héritages d'action publique et l'inertie de certains découpages territoriaux (en termes d'échelles ou de maille) influencent la mise en place de politiques publiques de paysage. Dans ces configurations, LOS_DAMA ! peut devenir une nouvelle arène actorielle permettant de décentrer les débats en raison de son caractère flexible et moins institutionnalisé. Le paysage est ici considéré comme outil de mobilisation des acteurs d'un territoire (Labat et Donadieu, 2013) et le projet européen permet de catalyser ce « momentum ».

34 À l'échelle locale, le programme est utilisé par les collectivités partenaires comme tribune, voire comme catalyseur de certains processus. Ainsi, Grenoble-Alpes- 
Métropole, dans le cadre du développement de son plan local d'urbanisme intercommunal, a pour projet une opération aménagement et programmation (OAP) paysage, dispositif relativement récent au sein des intercommunalités françaises. Dans ce contexte, l'intercommunalité a dédié l'une des activités pilotes du projet à un outil d'accompagnement à la mise en place de cette OAP par un volet d'éducation (master class à destination des élus locaux) et de monitoring (outil de suivi de l'OAP). Le projet Interreg sert alors de plate-forme de formalisation des politiques publiques et de sensibilisation des décideurs, y compris au sein des territoires voisins.

«LOS_DAMA! offre une valeur ajoutée pour accroître l'appropriation des composantes et des dynamiques paysagères, pour réexaminer les politiques locales sur leurs moyens d'action, pour associer les intercommunalités voisines et les PNR qui font face aux mêmes défis. » (PAP Grenoble, p. 7.)

S'il agit comme catalyseur en encourageant les coopérations interterritoriales et en modifiant le périmètre d'appréhension dans le cas grenoblois, le projet permet à Trente de questionner le lien aux politiques de planification spatiale et plus particulièrement le manque d'adaptation des plans réglementaires aux enjeux d'infrastructures vertes.

En outre, cette mise en politique publique peut pâtir de systèmes de planification et de modes de régulation politique hérités qui entravent la mise en place de projets interterritoriaux de paysage. Structurellement, les instances régionales ont un rôle faible dans les politiques publiques sur les terrains allemand et autrichien, en raison d'une concentration des compétences au sein des communes et des Länder. Si des embryons de structures intercommunales sont associés aux activités pilotes de Munich et de Vienne, elles ne disposent pas de compétences ad hoc et relèvent du registre d'associations de communes sous forme de coopération volontaire. La présence du projet est alors un levier pour faciliter une synergie entre acteurs qui ne disposent pas de la «bonne échelle» pour mener des projets. L'échelle intercommunale apparaît comme celle permettant la mise en cohérence d'approches sectorielles, même si les politiques associées sont également encadrées au niveau national (Moquay et al., 2007), voire influencées par le niveau européen, par incréments, comme dans le cadre des projets Interreg. À Vienne, l'absence de structure intercommunale mature s'ajoute à la frontière entre les Länder de Vienne et de Basse-Autriche qui reste assez étanche en raison du faible nombre de projets contractés entre la capitale et les communes voisines. Selon les acteurs du département Planification urbaine et utilisation des sols de la ville de Vienne et de l'association intercommunale Stadtumland Management, LOS_DAMA! a permis de faire converger des besoins et de les concrétiser: les communes de Gerasdorf et Bisamberg (Basse-Autriche) avaient émis la volonté d'être connectées à Vienne par un corridor écologique dans leurs documents stratégiques respectifs, tandis que la capitale souhaitait combler un vide dans sa ceinture verte. Le projet permet alors à ces territoires de réaliser un plan de paysage commun, chose inédite à l'échelle du pays.

\section{Unité d'action : la « mise en scène » avec les activités pilotes}

Les activités pilotes envisagées par les partenaires de projet et décrites dans le plan d'action pilote permettent d'identifier une convergence vers des formes de gouvernance collaborative et participative, qui différencient cependant les collectivités du projet dans les outils utilisés. 

local, de passer d'une culture sectorielle, techniciste, à une approche transversale par le paysage. Si à Grenoble, cette collaboration avec d'autres services est envisagée de façon partenariale comme appui réflexif à la mise en place des activités pilotes, dans le cas munichois ${ }^{8}$, les acteurs mettent à profit leurs liens avec des "personnes-clés » de départements connexes dans le cadre d'autres projets en s'en servant de façon informelle comme appui aux processus menés dans ce projet (entretien avec les collectivités membres du projet, Munich, 23 avril 2018). À l'échelle territoriale, la gouvernance collaborative peut être entravée par une asymétrie territoriale (Bertrand et al., 2015), entendue comme un traitement différencié (en termes de planification ou de politiques sectorielles) entre régions ou communes, qui questionne le rôle des associations de communes périurbaines dans la construction métropolitaine. Ainsi, sur l'une des activités pilotes de Munich menée conjointement avec l'association communale Munich Nord : « [Ses représentants] s'occupent d'une piste cyclable rapide vers Munich. Et le plus intéressant dans ces associations - y compris pour le Management régional de Munich Sud-Ouest, où la ville de Munich est également partie prenante, et présente dès la fondation - c'est qu'elles ont d'abord été conçues en opposition à la ville de Munich, et plus particulièrement Munich Nord. Il y avait en quelque sorte une alliance du Nord.» (Entretien avec les collectivités membres du projet, Munich, 23 avril 2018.)

39 Cette opposition renvoie à la fonction «utilitaire" du périurbain pour les villescentres, en termes d'accueil de grosses infrastructures qui menacent le paysage, sans qu'une contrepartie ne soit offerte quant à la représentativité de ces communes dans le projet métropolitain.

40 Enfin, les partenaires du projet, à l'exception de Salzbourg, font de la gouvernance participative soit un médium soit un objet à part entière de leurs activités pilotes, de sorte que la participation (à destination des habitants essentiellement) oscille entre simple information (Vienne), concertation (Trente) ou coconstruction d'un outil de cartographie participative (Munich et Grenoble).

\section{Conclusion}

41 L'articulation entre le projet transnational et les politiques existantes ou à venir au niveau local dans le domaine du paysage révèle le poids disparate des contextes nationaux et des cultures de planification. L'apport du transnational consiste ici, par une approche décentrée, à identifier les décalages entre politiques publiques et ambitions des acteurs locaux. Hormis ce rôle de facilitateur, le programme européen permet un apprentissage entre partenaires du projet sur des problématiques communes (entre Grenoble et Trente pour le périurbain dans la pente, entre Vienne et Munich pour la recherche d'instruments de coopération intercommunale).

Les convergences dans les modes de faire s'opèrent depuis les usages de ces paysages périurbains : si Grenoble, Trente et la région Piémont envisagent l'agriculture dans une perspective de justice alimentaire et environnementale, Vienne et Munich la traitent davantage dans sa relation aux usages récréatifs. Les partenaires de projet reconnaissent globalement l'importance de la réciprocité, qu'il s'agisse de mécanismes de compensation foncière (Salzbourg), financière (Munich) ou d'un travail partenarial interterritorial (Grenoble). Enfin, la région apparaît comme un niveau d'action ${ }^{9}$ et un

Projets de paysage, 19 | 2018 
périmètre optimal pour faire dialoguer les bonnes pratiques issues du local et les liens horizontaux à l'échelle transnationale.

Plus globalement, le projet LOS_DAMA ! questionne la recherche-action et les cultures partenariales. Au-delà d'oppositions entre une approche écologiste du paysage et une autre plus ancrée territorialement, le projet ne permet pas de questionner les catégories d'action publique utilisées, malgré leur appropriation très imparfaite par d'autres acteurs locaux: c'est notamment le cas du concept d'infrastructure verte, utilisé comme cadre normatif imposé d'en haut, par les politiques d'aménagement, mais peu exploité dans la pratique "quotidienne » des acteurs, sauf à des fins de communication. De même, hormis pour Trente et Grenoble, la dimension alpine n'est posée que comme décor, et la fabrique d'une périurbanité dans les Alpes reste peu étudiée, ce qui peut s'expliquer par le caractère composite de la définition du périurbain à l'échelle européenne.

L'auteure remercie Martina van Lierop (TU Munich), Corinna Jenal (université de Tübingen) et Guillaume Tournaire (Grenoble-Alpes Métropole) pour leur implication dans les plans d'action pilote ainsi qu'Emmanuel Roux pour ses remarques sur l'analyse lexicométrique. Cette recherche a bénéficié du soutien du projet LOS_DAMA!

\section{BIBLIOGRAPHIE}

Albert, C., Von Haaren, C., « Implications of Applying the Green Infrastructure Concept in Landscape Planning for Ecosystem Services in Peri-Urban Areas: An Expert Survey and Case Study ", Planning practice and research, vol. 32, n 3, 2014, p. 227-242.

Bertrand, N., Cremer-Schulte, D., Perrin, M., « Planification stratégique et asymétries territoriales. Grenoble et le Grand Genève, deux régions urbaines alpines à l'épreuve de la cohérence ", Journal of Alpine Research / Revue de géographie alpine, vol. 103, n³ 3, 2015, URL : http:// journals.openedition.org/rga/3104.

Bonin, S., Toublanc, M., Dérioz, P., Béringuier, Ph., « Des franges du projet urbain au projet de frange urbaine. La lisière comme nouvelle figure paysagère ? ", Projets de paysage, $\mathrm{n}^{\circ} 13$, janvier 2016, URL : http://www.projetsdepaysage.fr/fr/ des_franges_du_projet_urbain_au_projet_de_frange_urbaine\#.

Brand, C., « Modalités du retour des villes dans la gouvernance alimentaire. L'exemple de la région urbaine lyonnaise ", Urbanités, $\mathrm{n}^{\circ} 10,2018$.

Buijs, A. et al., « Assessment framework for urban green spaces governance arrangements : Research questions and methodology for Tier 2: Milestone 40 », 2015, URL : https:// greensurge.eu/working-packages/wp6/files/MS40__WP6_Tier_2_Methodology_and_research_questions_FINAL.pdf.

Bulkeley, H., « Reconfiguring environmental governance. Towards a politics of scales and networks ", Political geography, vol. 24, n 8, 2005, p. 875-902. 
Buyck, J., « Dessiner la campagne pour dessiner la ville », Projets de paysage, $\mathrm{n}^{\circ} 4$, juillet 2010, URL : http://www.projetsdepaysage.fr/fr/dessiner_la_campagne_pour_dessiner_la_ville\#.

Chéry, J.-P., « Les espaces périurbains en Europe : un grand écart entre description et prospective », Territoires 2040 : revue d'études et de prospective, 2010, p. 61-76.

Colomb, C., « The added value of transnational cooperation. Towards a new framework for evaluating learning and policy change ", Planning practice and research, vol. 22, n 3, 2007, p. 347-372.

Davodeau, H., « Les paysages, une nouvelle préoccupation dans la gestion des espaces périurbains ", Cahiers d'économie et de sociologie rurales, $n^{\circ} 77,2005$, p. 65-84.

Debray, A., « La trame verte et bleue, vecteur de changement des politiques de protection de la nature ou des politiques d'aménagement? », thèse de doctorat, université de Tours, 2016.

Di Leo, S., Salvia, M., « Local strategies and action plans towards resource efficiency in South East Europe », Renewable and sustainable energy reviews, vol. 68, 2017, p. 286-305.

Douence, H., Laplace-Treyture, D., « Les franges urbaines d'une ville moyenne : un paysage à cultiver ? Réflexions à partir de l'expérience paloise ", Projets de paysage, $n^{\circ} 13$, janvier 2016, URL : http://www.projetsdepaysage.fr/fr/ les_franges_urbaines_d_une_ville_moyenne_un_paysage_cultiver_\#.

Germaine, M-A., Temple-Boyer, E., Milian, J., Fourault-Cauët, V., Raymond, R., « La diversité des paysages des franges périurbaines : proposition d'indicateurs pour caractériser les espaces entre ville et campagne - deux cas de terrains franciliens ", L'Espace géographique, vol. 46, n 1, 2017, p. 19-40.

Görg, C., « Landscape Governance: The "politics" of scale and the "natural" conditions of places », Geoforum, vol. 38, n5, 2007, p. 954-966.

Guisepelli, E., Fleury, P., « Le paysage à la croisée des choix de développement local : Quelles connaissances pour quels enjeux ? Quels outils pour l'action?», dans Terrasson, D., BerlanDarqué, M., et Luginbühl, Y. (dir.), Paysages : de la connaissance à l'action, Versailles, Éditions Quae, 2007.

Haase, D., Frantzeskaki, N., Elmqvist, T., « Ecosystem services in urban landscapes. Practical applications and governance implications ", Ambio, vol. 43, n 4, 2014, p. 407-412.

Hansen, R., Rall, E.-L., « Green Surge Analytical framework milestone 34 », 2014, 33 p., URL : https://greensurge.eu/filer/MS34_Analytical_framework.pdf.

Hansen, R., et al., « Advanced urban green infrastructure planning and implementation: Innovative Approaches and Strategies from European Cities: Report 5.2 », 2016, URL : https:// greensurge.eu/working-packages/wp5/files/ D5_2_Hansen_et_al_2016_Advanced_UGI_Planning_and_Implementation_v3.pdf.

Labat, D., Donadieu, P., « Le paysage, levier d'action dans la planification territoriale », Espace géographique, vol. 42, $\mathrm{n}^{\circ}$ 1, 2013, p. 44-60.

Moquay, P., Aznar, O., Candau, J., Guérin, M., Michelin, Y., « Une typologie des interventions intercommunales en matière de paysage ", dans Terrasson, D., Berlan-Darqué M., et Luginbühl, Y. (dirs.), Paysages : de la connaissance à l'action, Versailles, Éditions Quae, 2007.

Poulot, M., « Du vert dans le périurbain. Les espaces ouverts, une hybridation de l'espace public (exemples franciliens) », EspacesTemps.net, 2013, URL : https://www.espacestemps.net/articles/ du-vert-dans-le-periurbain/. 
Poulot, M., Aragau, C., Rougé, L., « Les espaces ouverts dans le périurbain ouest francilien : entre appropriations habitantes et constructions territoriales ", Géographie Économie Société, vol. 18, $\mathrm{n}^{\circ}$ 1, 2016, p. 89-112.

Sgard, A., « Le paysage dans l'action publique : du patrimoine au bien commun », Développement durable et territoires, vol. 1, $\mathrm{n}^{\circ}$ 2, 2010, URL : http://journals.openedition.org/ developpementdurable/8565.

Sgard, A., Fortin, M.-J., Peyrache-Gadeau, V., « Le paysage en politique », Développement durable et territoires, vol. 1, $\mathrm{n}^{\circ}$ 2, $2010 \mathrm{URL}$ : http://journals.openedition.org/developpementdurable/8522.

Thomas, K., Littlewood, S., « From Green Belts to Green Infrastructure? The Evolution of a New Concept in the Emerging Soft Governance of Spatial Strategies ", Planning practice and research, vol. 25, n 2, 2010, p. 203-222.

Toublanc, M., Bonin, S., « Planifier les trames vertes dans les aires urbaines : une alliance à trouver entre paysagisme et écologie ", Développement durable et territoires, vol. 3, n² 2, 2012, URL : http://journals.openedition.org/developpementdurable/9347.

\section{NOTES}

1. Initié en novembre 2016 et qui prendra fin en octobre 2019.

2. Afin de faire un état des lieux des politiques publiques de paysage sur chaque terrain et de préciser le déroulement des activités pilotes mises en place localement et financées via le programme, ainsi que leur contribution à la création d'une infrastructure verte multifonctionnelle.

3. Définis par l'Agence comme des parcelles de terre végétalisées au cœur du tissu urbain, majoritairement à usage récréatif.

4. Espaces ouverts des zones périurbaines (dans un rayon de $50 \mathrm{~km} \mathrm{du} \mathrm{centre-ville)} \mathrm{qui}$ permettent de connecter les espaces verts urbains à la frange urbain/rural.

5. À noter que ni l'Allemagne ni l'Autriche n'ont ratifié cette convention.

6. Le plan d'action pilote étant conçu comme un document "vivant», évoluant au cours du projet, le tableau présenté ici exclut la région Piémont en raison d'éléments manquants.

7. Réalisée par le logiciel d'analyse qualitative Sonal, selon la méthode utilisée par Adèle Debray (2015) sur le discours des élus au sujet des trames vertes et bleues. Après lemmatisation (forme d'analyse lexicale) et exclusion des mots-outils ( «make», «but»...), le corpus rassemble plus de 14000 occurrences dont 2000 occurrences différentes (mots différents). Une sélection à partir de 5 occurrences a permis d'aboutir à la représentation graphique de la figure 4 .

8. Selon les acteurs impliqués dans le projet.

9. Niveau dans l'empilement des échelles de décision entre la commune et l'Union européenne.

\section{RÉSUMÉS}

Les espaces périurbains sont les terrains de nouvelles relations entre ville et campagne, où se réinventent les modes d'habiter. Sujets à une demande sociale, les paysages périurbains mêlent espaces ouverts et espaces bâtis. Les métropoles alpines constituent un terrain pertinent par leur 
proximité avec le grand paysage de montagne et la diversité des paysages "ordinaires ». Cet article apporte un retour réflexif sur un projet visant à créer un réseau de métropoles par les paysages et les infrastructures vertes. La diversité des terrains et le contexte interculturel questionnent les conditions de fabrication des politiques publiques de paysage, le «d'où l'on parle? » dans une perspective de recherche-action. On interrogera notamment la relation entre coopération transnationale et production de politiques de paysage situées dans un territoire donné et un contexte de planification parfois contraignant, pour réfléchir à la production de modes d'agir convergents sur le paysage.

Peri-urban spaces are areas where new relationships between the city and the countryside are being fostered and new lifestyles are being reinvented. Subjected to social demand, peri-urban landscapes combine open spaces and built-up areas. The choice of Alpine cities is relevant because of their proximity with the larger mountain landscape and the diversity of their "ordinary" landscapes. This article provides feedback on a project for the creation of a network of metropolitan areas combining landscapes and green infrastructures. The diversity of terrains and the inter-cultural context raise questions about how public landscape policies are defined, in the sense that these are conditioned by the respective positions of stakeholders in an action research perspective. We will analyse the relationship between transnational cooperation in defining landscape policies in a given territory and an urban planning context, which is sometimes restrictive, to reflect on how converging modes of action on the landscape can be defined.

\section{INDEX}

Mots-clés : paysage, action publique, périurbain, Europe, recherche-action

Keywords : landscape, public action, peri-urban, Europe, action research

\section{AUTEUR}

\section{AURORE MEYFROIDT}

Aurore Meyfroidt, docteure en géographie, est postdoctorante, UMR PACTE, université Grenoble Alpes, et chargée de projet Interreg Alpine Space, LOS_DAMA !

Aurore.meyfroidt[at]umrpacte[dot]fr 